Check for updates

Cite this: RSC Adv., 2019, 9, 38154

\title{
Magnetically targeted nanoparticles for imaging- guided photothermal therapy of cancer
}

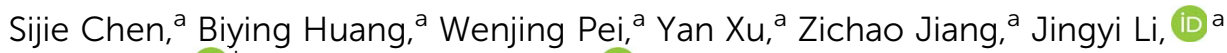 \\ Long Wang (iD ${ }^{\mathrm{b}}$ and Chengcheng Niu (D) *a
}

\begin{abstract}
Over the past several decades, nanocarriers have constituted a vital research area for accurate tumor therapy. Herein, magnetically targeted nanoparticles (IRFes) for photothermal therapy were generated by integrating IR780, a molecule with strong emission and absorption in the NIR spectrum and the ability to produce heat after laser irradiation, with $\mathrm{Fe}_{3} \mathrm{O}_{4}$ nanoparticles (NPs). These IRFes were guided to the tumor site by the application of an external magnetic field. In particular, the strong NIR absorption of IR780 was used for NIRF imaging, and we also demonstrated effective magnetic targeting for the photothermal ablation of tumors. In vitro cell viability and in vivo antitumor experiments showed that these IRFes can ablate 4T1 cells or transplanted 4T1 cell tumors when exposed to $808 \mathrm{~nm}$ laser irradiation and a magnetic field. In vivo experiments showed that IRFes only act on tumors, do not damage other organs and can be used to image tumors. These results demonstrate the enormous potential of local photothermal therapy for cancer under the guidance of external magnetic fields and reveal the prospect for the use of multifunctional nanoparticles in tumor therapy.
\end{abstract}

Received 11th October 2019

Accepted 18th November 2019

DOI: 10.1039/c9ra08281f

rsc.li/rsc-advances temperature is in the range of $37-41{ }^{\circ} \mathrm{C}$, PTT can accelerate blood flow and increase cell membrane permeability. Cancer cells can be selectively impaired at temperatures between 40 to $48{ }^{\circ} \mathrm{C}$, at which point harmful consequences such as protein folding and deformation, disruption of cellular membranes, increased sensitivity to radiotherapy and chemotherapy and irreversible damage occur. Within a short time (approximately 5 min), temperatures between 48 and $60{ }^{\circ} \mathrm{C}$ can trigger irreversible damage, serious and irreparable protein damage and DNA deformation and additional damage. Traditional hyperthermia therapy transmits heat to the tumor via radiofrequency radiation, ${ }^{18,19}$ ultrasound (US), ${ }^{20}$ or microwave. Laser-triggered tumor ablation has unique advantages over traditional tumor treatment methods including great controllability, limited damage to surrounding tissues, few systemic side effects, short recovery times and a commensurate reduction in hospitalization times, and visual in-process monitoring.

The effectual transmission of nanotheranostic agents to the target region is a necessary condition for cancer therapy. The passive targeting of some nanotherapeutics induced by enhanced permeability and retention (EPR) effects enable specific agent accumulation at the tumor site. In addition, targeting molecules conjugated to the surface of nanoparticles can recognize and bind to specific ligands of cancer cells to further enhance tumor treatment localization. ${ }^{21}$ For example, one of the typical superparamagnetic materials, $\mathrm{Fe}_{3} \mathrm{O}_{4}$, has been widely used for constructing magnetic targeted therapeutic systems. It has unique strengths; on the one hand, it is more controllable and predictable under the action of an external magnetic field,
${ }^{a}$ Department of Ultrasound Diagnosis, The Second Xiangya Hospital, Central South University, Changsha, Hunan 410011, China.E-mail: niuchengcheng@csu.edu.cn

${ }^{b}$ Department of Orthopedics, Xiangya Hospital, Central South University, Changsha, Hunan 410008, China 
and on the other hand, it can specifically deliver therapeutic agents, accumulate in high quantities in tumors and minimize the side effects in normal tissues. Additionally, both IR780 and $\mathrm{Fe}_{3} \mathrm{O}_{4}$ on NPs can absorb NIR light energy and convert it into heat.

Poly(lactide-co-glycolide) (PLGA) has attracted much attention because of its excellent biocompatibility and attractive loading capacity and has been developed as a nanocarrier for multifunctional therapeutics. Several materials that can interact with NIR light, such as graphene oxide, ${ }^{22}$ gold nanorods and small molecules, including indocyanine green (ICG) ${ }^{23}$ and IR780, ${ }^{24}$ which, upon discovery, were applied to the phototherapy of tumors. IR780, a lipophilic material with strong emission and absorption capacity in the NIR range and can produce heat by laser irradiation, is becoming the focus of cancer treatment and imaging. However, inferior aqueous solubility, rapid clearance and extremely low levels of uptake by tumors have severely restricted the clinical application of IR780. ${ }^{25,26}$ Some studies have proposed that IR780 be packaged into various nanomaterials to resolve these difficulties. ${ }^{27}$ In our study, we encapsulated IR780 into PLGA NPs, which has a core shell structure that can enhance antitumor efficacy by simultaneously encapsulating various hydrophobic and/or hydrophilic materials.

Herein, we report the magnetic targeting and photothermal diagnostic treatment with nanoparticles consisting of superparamagnetic iron oxide $\left(\mathrm{Fe}_{3} \mathrm{O}_{4}\right)$ and IR780-coated PLGA NPS (IRFes) on imaging and cancer treatment. Subsequently, PLGA nanoparticles were further demonstrated to have excellent biocompatibility and NIR-based imaging after magnetic targeting in vitro and in vivo. Moreover, effective photothermal ablation of tumors was achieved by utilizing the photothermal effect of IR780 that was generated by $808 \mathrm{~nm}$ NIR laser irradiation and the magnetic targeting induced by $\mathrm{Fe}_{3} \mathrm{O}_{4}$. Therefore, IRFes showed not only effective magnetic targeting of PTT to tumors but also NIRF imaging characteristics. Thus, IRFes, as effective therapeutic nanoagents, simultaneously showed great potential for NIR-imaging-guided diagnosis and magnetically targeted PTT under an external magnetic field.

\section{Materials and methods}

\subsection{Materials}

PLGA (lactide : glycolide $=50: 50, M_{\mathrm{w}}=10000 \mathrm{Da}$ ) and IR780 iodide and polyvinyl alcohol (PVA) were purchased from SigmaAldrich (USA). Oleic acid-treated $\mathrm{Fe}_{3} \mathrm{O}_{4}$ nanoparticles, with a mean diameter of $10 \mathrm{~nm}$, were purchased from Ocean Nano Tech Inc. (USA). Female BALB/c mice (6-8 weeks old and weighing $20 \mathrm{~g}$ ) were treated on the basis of the guidelines of the Department of Laboratory Animals, Central South University, China, and as approved by the Ethics Committee of the Second Xiangya Hospital, Central South University.

\subsection{Preparation of IRFes}

Simply, $100 \mathrm{mg}$ of PLGA and $3 \mathrm{~mL}$ of methylene chloride were mixed, and after stirring well, $3 \mathrm{mg}$ of IR780 and $0.2 \mathrm{~mL}$ of a $\mathrm{Fe}_{3} \mathrm{O}_{4}$ NP suspension ( $30 \mathrm{mg}$ Fe per $\mathrm{mL}$ ) were added into the mixture in order, then $15 \mathrm{~mL}$ of $4 \% \mathrm{w} / \mathrm{v}$ cold PVA solution were added and emulsified by an ultrasonic processor at a power of $130 \mathrm{~W}$ and frequency of $20 \mathrm{kHz}$ for $2 \mathrm{~min}$. The resulting emulsion was added to $20 \mathrm{~mL}$ deionized water and stirred at room temperature (RT) until the methylene chloride became volatile. Finally, the created NPs were centrifuged at $12000 \mathrm{rcf}$ for $7 \mathrm{~min}$ and washed three times with deionized water. All operations were performed in the dark.

\subsection{Characterization}

First, transmission electron microscopy (TEM, Hitachi H-7600, Japan) was used to affirm the existence of $\mathrm{Fe}_{3} \mathrm{O}_{4}$ particles in the NP shells. The size and surface charge was detected by a Malvern size analyzer (Malvern Nano $\mathrm{ZS}$, UK), the IRFes was dispersed in PBS and the concentration was $1 \mathrm{mg} \mathrm{mL}^{-1}(\mathrm{pH}$ 7.4). To explore the magnetic features, we set a magnet next to a glass vial filled with an IRFes solution. The IRFes were injected at one end of a hose ( $1 \mathrm{~mm}$ inner diameter) and were collected at the other end. The injection flow rate was approximately 50 $\mathrm{mL} \min ^{-1}$ to simulate the intravascular fluid state of the tumor. A magnetic attractor was applied to one side of the hose to observe the response of the nanoparticles to a magnetic field. A Cary 5000 UV-vis-NIR spectrophotometer (USA) was used to detect the absorption spectra of the IRFes and verify the existence of IR780. The IR780 encapsulation and loading efficiencies were calculated according to the following formulas:

$$
\begin{gathered}
\text { Encapsulation efficiency }(\%)=W_{\mathrm{E}} / W_{\mathrm{t}} \times 100 \% \\
\text { Loading efficiency }(\%)=W_{\mathrm{E}} / W_{\mathrm{T}} \times 100 \%
\end{gathered}
$$

where $W_{\mathrm{E}}$ is the totality of all the IR780 molecules encapsulated in the IRFes, $W_{\mathrm{t}}$ is the totality of all the input IR780, and $W_{\mathrm{T}}$ is the weight of the PLGA NPs.

\subsection{Colloidal stability of IRFes}

The nanoparticles were resuspended in PBS or 10\% fetal bovine serum (FBS) at a concentration of $5 \mathrm{mg} \mathrm{L}^{-1}$. The same amount of suspension was dispensed into several centrifuge tubes and allowed to stand in a cell culture incubator at $37^{\circ} \mathrm{C}$. A centrifuge tube containing sample was randomly selected for the continuous detection of nanoparticle hydrodynamic size and zeta potential using a Malvern size analyzer.

\subsection{Thermal stability and photostability of IRFes}

To study the thermal stability of the nanoparticles, $1 \mathrm{~mL}$ of $2 \mathrm{mg}$ $\mathrm{mL}^{-1}$ IRFes and indocyanine green (ICG) were subjected to cycles of $808 \mathrm{~nm}$ laser irradiation with 4 ON/OFF repeats - laser irradiation (ON, $3 \mathrm{~min}$ ) and gradual cooling (OFF, $10 \mathrm{~min}$ ); the temperature of the solution was detected every $30 \mathrm{~s}$, and the IR780 concentration was $0.016 \mu \mathrm{mol} \mathrm{mL}{ }^{-1}$, which was the same as that of ICG.

The photostability of the IRFes and IR780 was analyzed by NIR fluorescence imaging. Specifically, $0.2 \mathrm{~mL}$ of IRFes or IR780 was placed in a 24 -well plate at physiological temperature $\left(37^{\circ} \mathrm{C}\right)$, and 
the $24 \mathrm{~h}$ fluorescence image was captured after stimulation with an excitation wavelength of $790 \mathrm{~nm}$. A Lumina IVIS Spectrum imaging system (PerkinElmer, USA) was used to analyze the images to accurately quantify the fluorescence signal.

\subsection{In vitro PTT effect}

One milliliter of IRFes, IR780, $\mathrm{Fe}_{3} \mathrm{O}_{4}$ NPs, the mixture of free IR780 and $\mathrm{Fe}_{3} \mathrm{O}_{4}$ NPs, phosphate buffered saline (PBS), and phthalocyanine green (ICG) were poured into an Eppendorf tube respectively and irradiated with an $808 \mathrm{~nm}$ laser at an intensity of $1.0 \mathrm{~W} \mathrm{~cm}^{-2}$ for $5 \mathrm{~min}$. ICG and PBS were considered the positive and negative control, respectively. The concentration of the IRFes was $2 \mathrm{mg} \mathrm{mL}{ }^{-1}$. The concentration of IR780

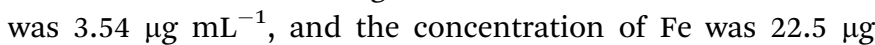
$\mathrm{mL}^{-1}$. The molar volume of ICG was $0.016 \mu \mathrm{mol} \mathrm{mL} \mathrm{mL}^{-1}$, which was equal to that of IR780 in our experiment. The temperature was determined using an infrared thermal imaging camera (FLIR C2, USA). Then, $1 \mathrm{~mL}$ of each IRFes concentration $(0,0.5$, 1.0 and $2.0 \mathrm{mg} \mathrm{mL}^{-1}$ ) was placed under the $808 \mathrm{~nm}$ laser, and the temperature was recorded every $30 \mathrm{~s}$.

\subsection{Cell experiments}

Cell cytotoxicity induced by IRFes exposure was measured by MTT assay. ${ }^{28}$ Mouse breast cancer $4 \mathrm{~T} 1$ cells were plated $1 \times 10^{4}$ cells per well on a 96-well plate for $12 \mathrm{~h}$ at $37{ }^{\circ} \mathrm{C} 5 \% \mathrm{CO}_{2}$ in an incubator. Different concentration of IRFes $(0.2,0.4,0.6$ and $0.8 \mathrm{mg} \mathrm{mL} \mathrm{m}^{-1}$ ) or without IRFes were added to the cells and cultured for $6 \mathrm{~h}$. At the same time, the magnet targeting group were treated with four round magnets (diameter $=1.0 \mathrm{~cm}$, maximum magnetic field strength $=6.0 \mathrm{Gs}$ ) under the four corners of each 96-well plate in the first $2 \mathrm{~h}$. Then, subjected to laser light at $1.0 \mathrm{~W} \mathrm{~cm} \mathrm{~cm}^{-2}$ for $5 \mathrm{~min}$. The strengths of the magnetic fields were reduced with distance. The depth of the liquid in each well was $3 \mathrm{~mm}$, and the bottom thickness of 96well plate was $1 \mathrm{~mm}$. The magnetic field strength was $5.2 \mathrm{Gs}$ $1 \mathrm{~mm}$ away from the magnet. The magnetic field strength was $4.7 \mathrm{Gs}$ at a distance of $4 \mathrm{~mm}$. Finally, the cell viability after each different treatment was calculated.

The level of IRFes phagocytosed was investigated by confocal laser scanning microscopy (CLSM, Zeiss LSM 510) to further evaluate the magnetic targeting efficacy. Medium $(0.1 \mathrm{~mL})$ with $0.2 \mathrm{mg} \mathrm{mL}^{-1}$ IRFes was added to $4 \mathrm{~T} 1$ cells. The abovementioned magnets were placed under the dish with cells and allowed to stand for $1 \mathrm{~h}$. The 4T1 cells not subjected to a magnet field were used as controls. The dish thickness was approximately $1 \mathrm{~mm}$, and the liquid depth in the dish was $3 \mathrm{~mm}$. All cells were washed thoroughly 3 times with PBS and dyed with 4,6diamidino-2-phenylindole (DAPI).

To directly inspect photothermal efficiency, the 4T1 cells were stained with propidium iodide (PI), for dead cells, and DAPI, and the observations were analyzed by cell treatment groups: (I) laser irradiation, (II) IRFes, (III) IRFes with laser irradiation, (IV) IRFes with laser irradiation and magnetic targeting were stained with PI and DAPI. The concentration of IRFes was $0.2 \mathrm{mg} \mathrm{mL}^{-1}$. The cells were subjected to the magnet for $2 \mathrm{~h}$ and then irradiated with a laser of $1 \mathrm{~W} \mathrm{~cm}^{-2}$ for $5 \mathrm{~min}$.
The cells were examined using an inverted fluorescence microscope (DMIL, Leica, Japan) to distinguish any dead cells.

\subsection{Fluorescence imaging}

A Lumina IVIS Spectrum imaging system (PerkinElmer, USA) was used to obtain the in vitro fluorescence images $\left(\lambda_{\mathrm{ex}}=\right.$ $790 \mathrm{~nm}$ and $\lambda_{\mathrm{em}}=810 \mathrm{~nm}$ ). IRFes and $\mathrm{Fe}_{3} \mathrm{O}_{4} @$ PLGA NPs (without IR780) in the presence of different concentrations of IR780 $\left(10,20,40\right.$ and $\left.80 \mu \mathrm{g} \mathrm{mL} \mathrm{m}^{-1}\right)$ were suspended in 24-well plates.

Female BALB/c mice (6-8 weeks old and weighing $20 \mathrm{~g}$ ) were purchased from the Laboratory Animal Center of Central South University (China) and received care in compliance with the rules of the Department of Laboratory Animals, Central South University, China. All procedures were approved by the Ethics Committee of the Second Xiangya Hospital, Central South University, China. When in the logarithmic growth phase, the $4 \mathrm{~T} 1$ cells $\left(1 \times 10^{6}\right)$ were digested and injected into the right flank of the mice by subcutaneous injection to establish a breast cancer model, and the volume of tumor reached $100 \mathrm{~mm}^{3}$ after one week.

To obtain in vivo fluorescence images, a Lumina IVIS spectrum imaging system (PerkinElmer, USA) was also used to visualize The $200 \mu \mathrm{L}$ pf IRFes $\left(10 \mathrm{mg} \mathrm{mL}^{-1}\right)$ that were injected into the tail vein of the mice from 2 groups $(n=5)$ : (1) with magnetic targeting and (2) without magnetic targeting. To detect the in vivo magnetic targeting effect of IRFes, a magnet with a maximum magnetic field strength of 40.6 Gs and magnetic fields that were gradually attenuated with distance were applied next to the tumor for $4 \mathrm{~h}$. The maximum magnetic field strength was 34.2 Gs when the distance was $3 \mathrm{~mm}$ away from the magnet. In each group, the depth of the tumor was approximately $5 \mathrm{~mm}$. Subsequently, important organs and tumors were imaged, and the fluorescence data were analyzed.

\subsection{In vivo antitumor therapy}

When the tumor volume reached $60 \mathrm{~mm}^{3}$, the tumor-bearing mice were randomly separated into five groups $(n=5$ per group): (1) saline, (2) IRFes, (3) laser irradiation, (4) IRFes with laser irradiation, and (5) IRFes with laser irradiation and magnetic targeting. They were injected with different treatments intravenously. All NPs were administered at a concentration of $10 \mathrm{mg} \mathrm{mL} \mathrm{mL}^{-1}$, and the IR780 concentration was $0.177 \mathrm{mg} \mathrm{mL} \mathrm{m}^{-1}$. A magnet with a maximum magnetic field strength of 40.6 Gs was placed in group (5) close to the tumor $24 \mathrm{~h}$ after injection, where it remained for $4 \mathrm{~h}$ and was exposed to a $808 \mathrm{~nm}$ laser of $1.0 \mathrm{~W} \mathrm{~cm}^{-2}$ for $5 \mathrm{~min}$. Temperature detection was performed with an infrared thermal imaging camera (FLIR C2, USA) every $30 \mathrm{~s}$, and a time-temperature curve was generated. Tumor volume and mouse body weight were measured every other day after photothermal treatment for 14 days, and tumor growth and mouse body weight curves were plotted. On the 14th day, the mice were sacrificed through use of excessive pentobarbital sodium, the tumor tissues were removed, and the tumor volume was measured to evaluate the antitumor effect of the treatments in all the groups. Tumor 
tissues and important organs (heart, liver, spleen, lung, and kidney) were used to create pathological slides and stained with hematoxylin and eosin (H\&E). Analyses of tumor tissue apoptosis and proliferation were performed using TUNEL and Ki-67 immunofluorescence staining.

\section{Results and discussion}

\subsection{Characterization}

The IRFes emulsion was successfully prepared, and it appeared uniformly green. A TEM image showed that the IRFes had smooth and uniformly spherical shapes that showed the presence of iron particles, as indicated by many black particles that were proportionally well embedded into the spherical shell (Fig. 1a). A dynamic light scattering (DLS) system detected that the size distribution of the IRFes was almost symmetrical, and the mean diameter was $334 \mathrm{~nm}(\mathrm{PDI}=0.042)$ (Fig. 1b). The surface zeta potential was $-1.55 \mathrm{mV}$ (Fig. 1c).

To check the magnetization of the IRFes, we added an additional magnetic field. After $3 \mathrm{~min}$, a large number of nanoparticles in PBS had gathered in the direction of the magnet, and almost all of them were aggregated after $1 \mathrm{~h}$, indicating that these nanoparticles display remarkable magnetic responsiveness (Fig. 1d). Furthermore, as shown in Fig. 1e, the nanoparticles in the flowing state of the tube were also attracted by the magnet to the magnetic field side, and many black nanoparticles could be seen in the magnetic field side after $60 \mathrm{~s}$, demonstrating that the nanoparticles would have superior magnetic responsiveness in the fluid of the tumor blood vessels and were unchanged in the liquid state.

The absorption spectra of IRFes $\left(10 \mathrm{mg} \mathrm{mL}^{-1}\right)$ with the various components are shown in Fig. 1f. IR780 with an encapsulation efficiency of approximately $48.26 \pm 2.11 \%$ and a loading efficiency above $1.77 \%$. The Fe content was $92.28 \pm$ $3.20 \mu \mathrm{g} \mathrm{mL}^{-1}$. The spectrum of the PLGA NPs had a straight line at $400-900 \mathrm{~nm}$, indicating that they did not absorb light.

The absorbance curves of the $\mathrm{Fe}_{3} \mathrm{O}_{4}$ NPs at $400-900 \mathrm{~nm}$ were oblique and without an obvious peak, which indicated that they had some ability to absorb light, while the free IR780 had a high and sharp peak at 780-790 $\mathrm{nm}$, which indicated strong absorption and emission capabilities in the near-infrared region. However, the absorption peak of the IRFes, obtained by UV spectrometry, showed a redshift of approximately $795 \mathrm{~nm}$ compared with the absorption peak of $780 \mathrm{~nm}$ for the free IR780, which was attributed to the introduction of the auxiliary color group, $\mathrm{Fe}_{3} \mathrm{O}_{4}$, affirming the successful loading of IR780 and $\mathrm{Fe}_{3} \mathrm{O}_{4}$ onto the IRFes. These findings indicate that IRFes are excellent photoabsorbing agents in the near-infrared region.

\subsection{Colloidal stability of IRFes}

The IRFes were resuspended in PBS or 10\% FBS for 7 days, and the size and zeta potential were determined every day by a Malvern size analyzer used to study colloidal stability. The size distribution measured by a dynamic light scattering (DLS) system was neither larger nor smaller in PBS compared to the distribution in $10 \%$ FBS (Fig. 2a). In addition, the zeta potential changed little over time (Fig. 2b), revealing that the NPs had outstanding colloidal stability. Hence, it was highly anticipated that the stability of the NPs after intravenous injection would have prolonged circulation in vivo.

\subsection{Thermal stability and photostability of IRFes}

The thermal stability of the IRFes was studied by comparing the IRFes to the traditional PTT reagent ICG, which has been approved by the US Food and Drug Administration. As shown in
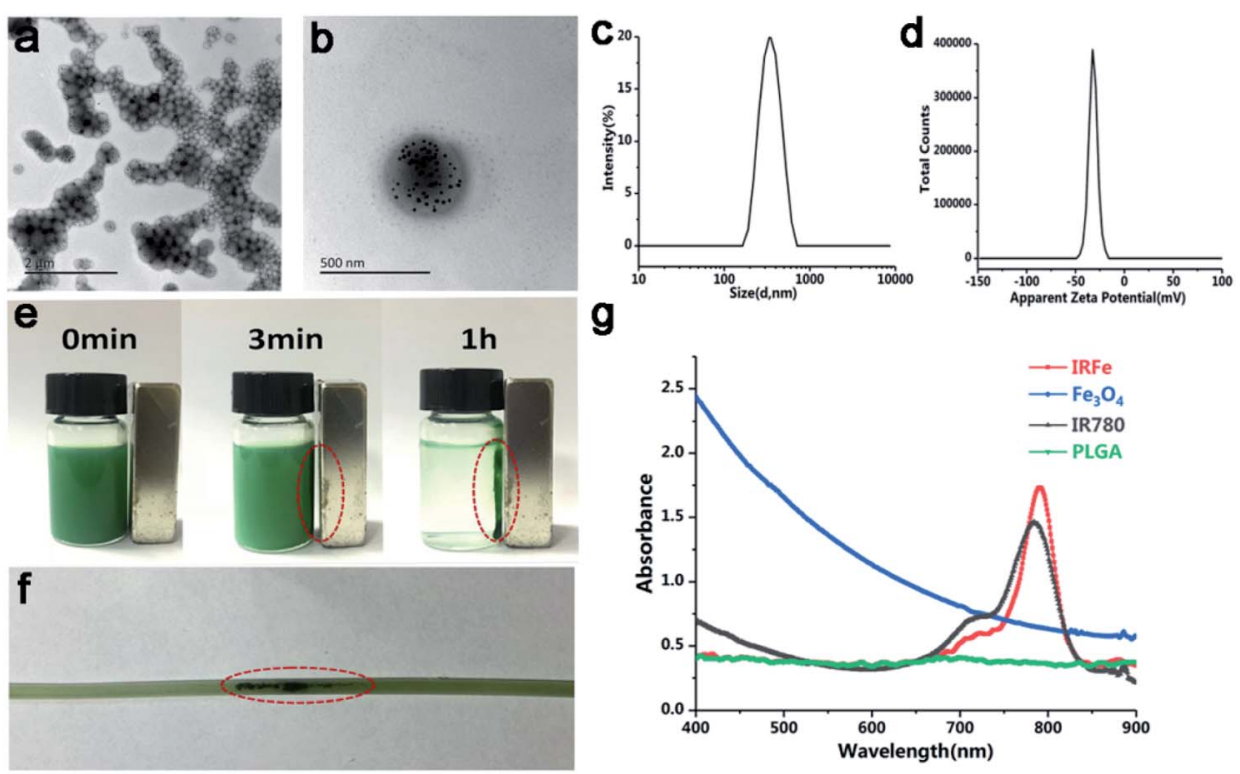

g

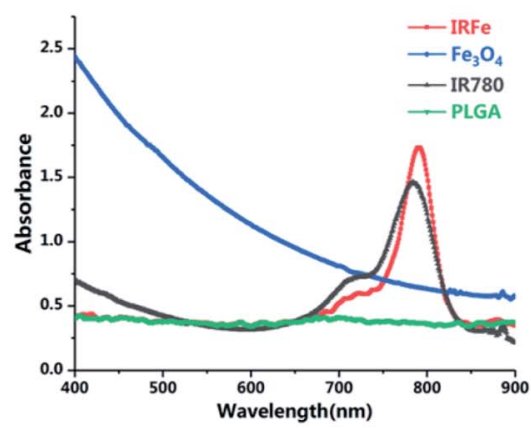

Fig. 1 (a and b) TEM images ((a) 9600x; scale bar, $2 \mu \mathrm{m}$; (b) $46000 \times$; scale bar, $500 \mathrm{~nm}$ ), (c) size and (d) zeta distributions of IRFes. (e and f) Photograph of IRFes solution (10 $\mathrm{mg} \mathrm{mL}^{-1}$ ) in a vail (e) and hose (f) with an external magnetic field. (g) UV-vis-NIR absorption spectra of Fe $\mathrm{O}_{4}$, IR780, PLGA NPs, and IRFes. 

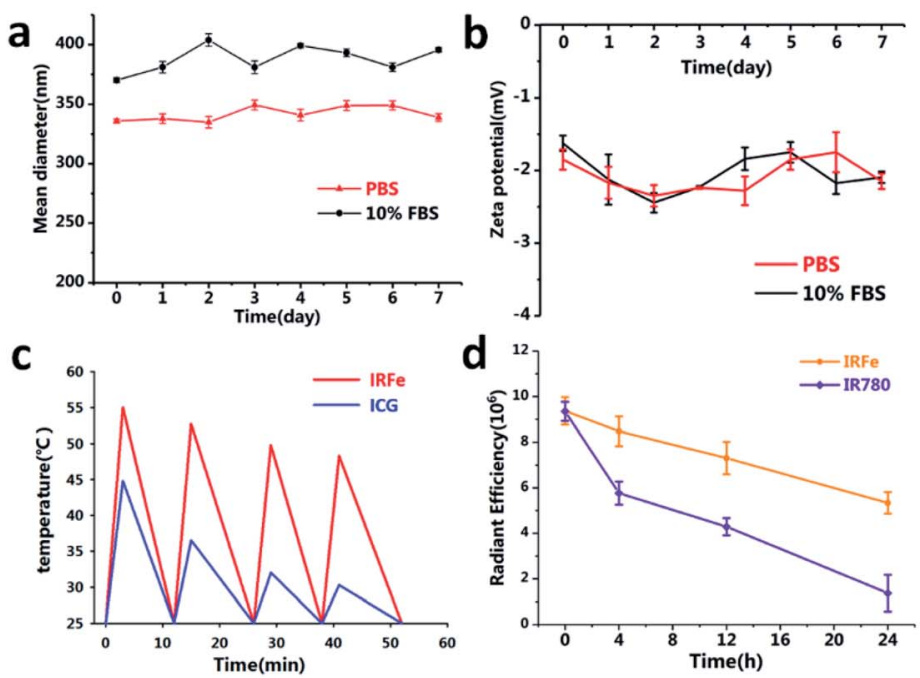

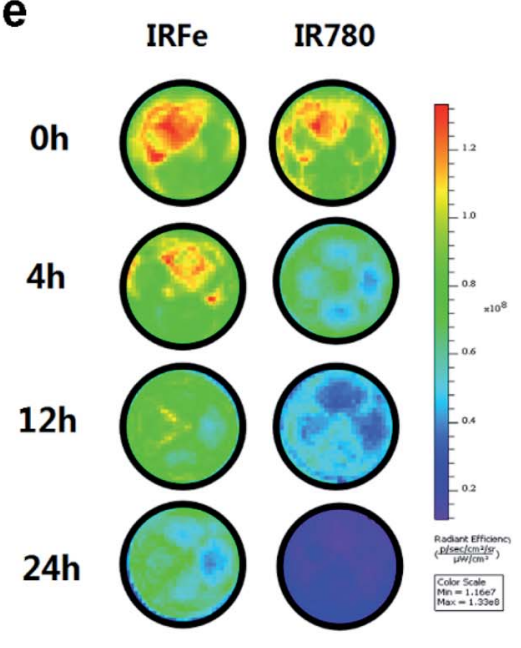

Fig. 2 (a and b) Size and zeta distributions of IRFes in $1 \times$ PBS or $10 \%$ FBS for 7 days. (c) Photostability of IRFes irradiated with a NIR 808 nm laser $\left(1.0 \mathrm{~W} \mathrm{~cm}^{-2}\right.$ ) comparing with ICG. ( $\mathrm{d}$ and e) Comparison of the fluorescence stability of IR780 and IRFes within $24 \mathrm{~h}$ by NIR fluorescence images.

Fig. 2c, the maximum temperature of the IRFes increased to 55.0, 52.7, 49.8 and $47.3{ }^{\circ} \mathrm{C}$, and the ICG temperatures were $44.8,36.5,32.0$, and $30.3{ }^{\circ} \mathrm{C}$ in the 4 laser $\mathrm{ON} / \mathrm{OFF}$ cycles. Notably, IRFes could increase beyond $42{ }^{\circ} \mathrm{C}$ after 4 cycles of laser irradiation, which hinted that the IRFes can be efficient PTT agents. Nevertheless, the effect on the free ICG was diminished, and the temperature decreased below $42{ }^{\circ} \mathrm{C}$. Therefore, the IRFes have become promising therapeutic agents for tumors, relying on their preeminent PTT properties and prominent light photostability.

In addition, the poor stability in aqueous solutions, inferior photodegradation and high levels of thermal degradation led to the cessation of IR780 iodide use in clinical applications as an NIR tracer. ${ }^{25,29}$ Some studies have been dedicated to encapsulating IR780 iodide into a variety of nanomaterials to overcome those shortcomings. ${ }^{25,26,29}$ To determine whether PLGA has the ability to defend IR780 from degradation, we measured alterations in the near-infrared fluorescence signal of IR780 and the IRFes over time. The NIR fluorescence signal from IR780 and the IRFes gradually declined with time (Fig. 2d and e). However, the signals from the IRFes demonstrated a much slower decline than that shown by IR780 at different time points, namely, 0, 4, 12 , and 24 hours. Notably, compared to pure IR780 in solution, IRFes encapsulating IR780 into the PLGA shell seemed to effectively prevent the internal IR780 from degrading and enhance its long-term stability. IRFes have been used for all subsequent studies on the basis of their stable physicochemical and photothermal properties.

\subsection{In vitro PTT effect}

The temperature changes after irradiation are shown in Fig. 3a and $b$. The point at which the IRFes maximum temperature reached $55{ }^{\circ} \mathrm{C}$ was greatly increased as it was for the mixture of IR780 and $\mathrm{Fe}_{3} \mathrm{O}_{4} \mathrm{NPs}$, for which the maximum temperature was $53.3{ }^{\circ} \mathrm{C}$. Likewise, the maximum temperature increases were observed for IR780, and ICG at 50.5 and $48.2{ }^{\circ} \mathrm{C}$, respectively. Moreover, compared to other components, the temperature increase of the $\mathrm{Fe}_{3} \mathrm{O}_{4}$ NPs was gradual and steady to $38.2{ }^{\circ} \mathrm{C}$. Compared to the ICG response to laser irradiation, IR780 was more sensitive and rapid, with a steep temperature curve that increased more rapidly. Due to the superposition of the heating effect of IR780 and the $\mathrm{Fe}_{3} \mathrm{O}_{4}$ NPs, the temperatures of IRFes were slightly higher than that of IR780 individually and showed almost the same PTT efficiency and efficacy as that of IRFes, which further proved that the PLGA shell rarely affected the PTT effect. Notably, the highest temperatures for $0,0.5,1.0$, and $2.0 \mathrm{mg} \mathrm{mL}{ }^{-1}$ IRFes were 25.4, 35.2, 42.2, and 55.0 ${ }^{\circ} \mathrm{C}$ (Fig. 3c), respectively, indicating that the PTT properties of the IRFes were significantly concentration-dependent. The photothermal conversion efficiency $(\eta)$ was evaluated by $808 \mathrm{~nm}$ laser irradiation $\left(1.0 \mathrm{~W} \mathrm{~cm}^{-2}\right)$ of $1.0 \mathrm{mg} \mathrm{mL}^{-1}$ IRFes, according to our previous study. ${ }^{30}$ The linear regression curve between $\ln (\theta)$ and the cooling time could be used to infer $\tau$, and the $\eta$ value of the IRFes was determined to be $37.5 \%$ (Fig. 3d).

\subsection{In vitro antitumor activity}

Low cytotoxicity is the most vital feature of an in vivo tracer. The cell viability that was determined without the presence of IRFes was not significantly reduced regardless of the exposure to laser irradiation (Fig. 4a, $p>0.05$ ). No apparent toxicity was observed, and the dose-dependent 4T1 cell viability remained above $90 \%$, even at concentrations as high as $0.8 \mathrm{mg} \mathrm{mL}^{-1}(p>0.05)$. The results showed no explicit relationship between IRFes concentration and cell viability, suggesting rare NP-induced cytotoxicity, significantly supporting their further use in vivo. At the same IRFes concentrations, the cell viability of the NIRirradiated group was significantly reduced compared to that of the unirradiated group, indicating a favorable photothermal cell toxicity of the NPs $\left({ }^{*} p<0.05\right)$. In addition, compared to nonmagnetic targeting, magnet targeting attracted more IRFes 


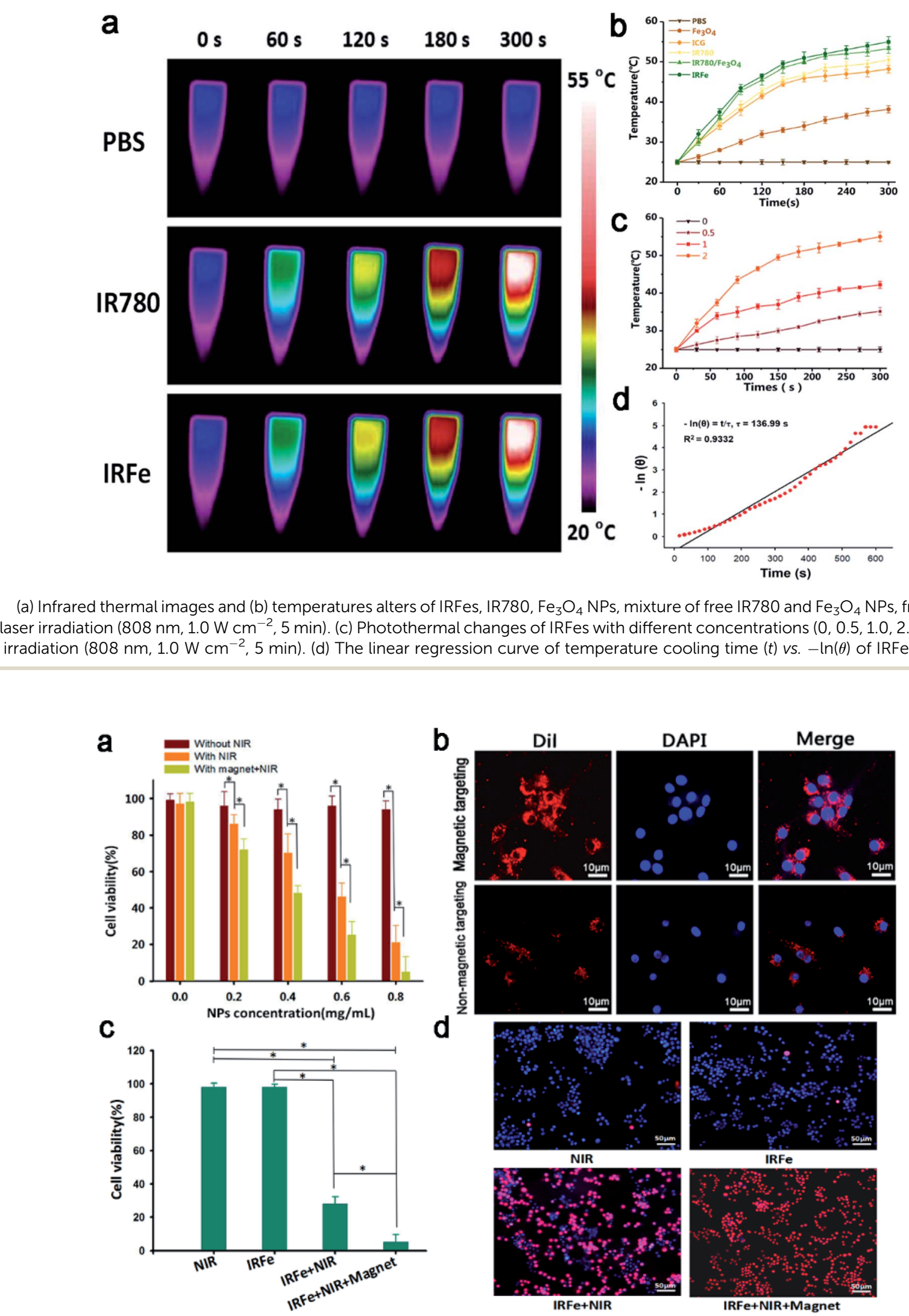

Fig. 4 Cell experiments. (a) Relative viability of 4T1 cells incubated with different concentrations of IRFes under laser irradiation (808 $\mathrm{nm}$, $1 \mathrm{~W}$ $\mathrm{cm}^{-2}, 5 \mathrm{~min}$ ). (b) Fluorescence images of $4 \mathrm{~T} 1$ cells incubated with IRFes with or without magnetic targeting. (c) Relative viability and (d) DAPI/PI costained 4T1 cells after different treatments: (I) laser irradiation; (II) IRFes; (III) IRFes with laser irradiation; (VI) IRFes with laser irradiation and magnetic targeting (scale bar, $10 \mu \mathrm{m}$ ). 
to the cells, where they firmly remained and were phagocytized, leading to an observable decrease in cell viability $(* p<0.05)$ and indicating that the binding of magnetically targeted IRFes for PTT greatly lowered cell viability.

Then, the phagocytosis assay of the IRFes labeled with red fluorescence DiI dye was performed using CLSM. As shown in Fig. 4b, the DiI fluorescence of the magnet targeting group was significantly brighter than that of the group without magnetic targeting, as magnetic targeting greatly promoted the aggregation and endocytosis ability of the IRFes.

Next, we investigated the in vitro photothermal effect of the IRFes in 4T1 cells. As shown in Fig. 4c and d, the cytotoxicity of only the IRFes group and only the laser group was exceedingly low, indicating that they had almost no ability to kill cells alone. However, once the IRFes group was exposed to the $808 \mathrm{~nm}$ laser, the number of dead cells increased significantly and the cell viability was reduced to $28 \%(* p<0.05)$, as the IR780 of the IRFes converted the absorbed $808 \mathrm{~nm}$ laser into heat and burned numerous cells. Particularly, when adding an external magnetic field, the cell viability of group IV was as low as $5 \%(* p$ $<0.05$ ), which again proved the outstanding magnetic targeting of IRFes and accelerated their aggregation to the cells.

\subsection{Fluorescence imaging}

The NIRF images and fluorescence SI of the IRFes at different concentrations at room temperature $\left(25^{\circ} \mathrm{C}\right)$ are shown in Fig. 5a and $\mathrm{b}$. Without IR780, the IRFes had no detectable fluorescence signal; however, as the concentration of IR780 in the IRFes was increased, the NIRF signal intensity also gradually increased. These findings indicate that the IRFes possess brilliant and remarkable NIRF properties, which makes them advanced NIR tracers for tumors.

Studies have shown that the addition of a magnetic field mediates specific site targeting and is an effective targeting method. ${ }^{31}$ Based on the DIR-labeled IRFes, we studied the distribution of the IRFes in mice by imaging both in vivo and ex vivo organs. By observing and detecting the DIR fluorescence, we could compare the differences between the distribution and duration of the IRFes in various organs and, in particular, tumor tissues. The mice were intravenously injected and divided into 2 groups: one with magnetic targeting and the other without magnetic targeting. As shown in Fig. 5c, when magnetic targeting was added, the fluorescence intensity of the tumor was obviously stronger than that of the group without magnetic targeting. Moreover, the enhanced signals were maintained in the tumor for as long as $24 \mathrm{~h}$, which indicates that with the added magnetic mediation, more IRFes were targeted to the tumor site. Then, we imaged and quantified the $e x$ vivo organs and tumors. The NIRF images and the fluorescence SI are shown in Fig. 5d and e; the early high concentration accumulation in the lung and liver met expectations because the macrophage system was expected to clear foreign matter within $24 \mathrm{~h}^{32}$ In addition, a tremendous increase in tumor fluorescence was observed in the magnetic targeting group, in contrast to that observed in the group without magnetic
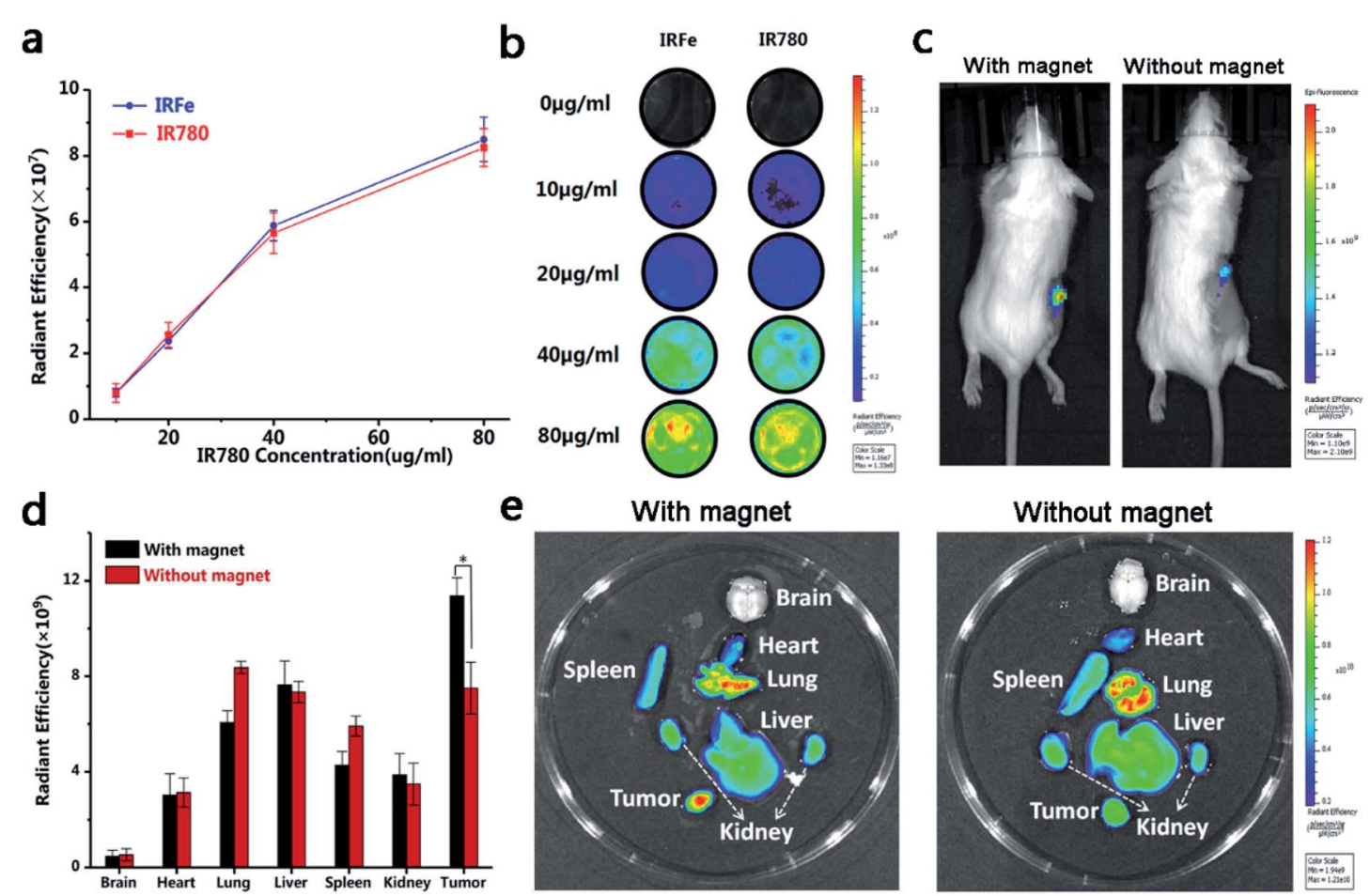

e
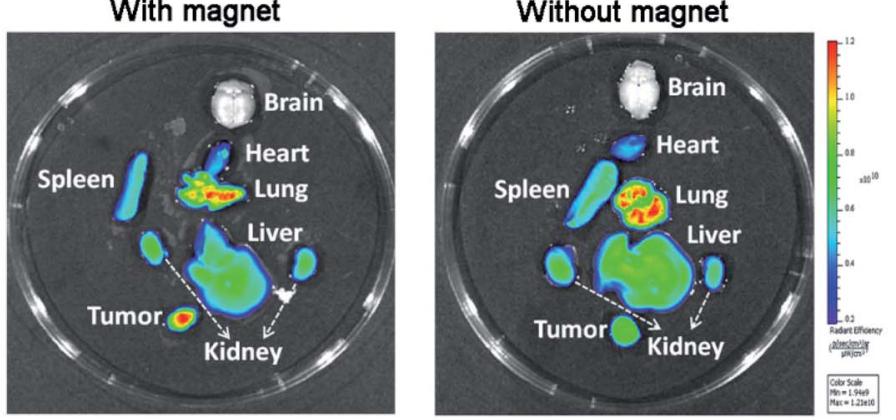

Fig. 5 In vitro NIR fluorescence images (a) and plot for fluorescence signal intensity (b) of IR780 and IRFes. (c) In vivo NIRF imaging for tumors with or without magnetic targeting at $24 \mathrm{~h}$ after IRFes administration. (d) Averaged NIRF signals of major organs and tumors. The difference is statistically significant. (e) Ex vivo NIRF images of major organs and tumors in tumor-bearing mice. The difference is statistically significant (* $p<$ 0.05). 
targeting, nevertheless, no significant changes were found in other organs. This phenomenon was related to the EPR (enhanced permeability and retention effect). The microvascular endothelium in normal tissues is dense and structurally intact such that macromolecules and lipid particles cannot easily penetrate the vessel wall, while solid tumor tissue has wide gaps in the blood vessel wall, incomplete structure, and lack of lymphatic reflux, resulting in selective high permeability and retention of macromolecular substances and lipid particles. The nanocarrier macromolecules were concentrated in the tumor region through the EPR effect in a passively dispersed manner, where they played a passive targeting role.

In addition, although the EPR takes effect relatively slowly, while nanocarriers enter the tumor site, it is rigorously maintained over time and regardless of concentration. ${ }^{17}$ Under the dual targeting effects of magnetic attraction and EPR, the intracellular fluorescence intensity of the magnetic targeting group was higher than that of the nonmagnetic targeting group. These results indicate that magnetic targeting is conducive to the accumulation of magnetic NPs in the direction of tumors, making them potential therapeutic reserve forces for prospective combination therapies of tumors.

\subsection{In vivo cytotoxicity}

For further use of IRFes as magnetic targeting photothermal therapy agents, it is necessary to clearly discern their potential for inducing toxicology in vivo. Consequently, a toxicity evaluation of the IRFes in vivo, including the biodistribution and histological analyses and body weight measurements. BALB/c tumor-bearing mice were intravenously injected with a dose of IRFes $\left(200 \mu \mathrm{L}, 10 \mathrm{mg} \mathrm{mL}{ }^{-1}\right)$. First, biodistribution was determined, as described in the previous section, through fluorescence imaging (Fig. 5c-e). Second, the histological detection of the major organs (heart, liver, spleen, lung and kidneys) stained with hematoxylin and eosin (H\&E) of the mice 14 days after injection with IRFes showed no notable inflammatory lesions or organ damage for all major organs, compared with these conditions in the control mice. Most important, no necrosis was found for any group (Fig. 6a). Third, body weight undulation is a reference index for inspecting the toxicity of IRFes. No significant body weight loss was observed for any of the experimental groups over a 14 day period (Fig. 6b), proving that a given dose of IRFes rarely induced toxicity in vivo and that IRFes can serve as therapeutic agents by injection in vivo.

\subsection{In vivo anticancer efficacy}

On the basis of the outstanding in vitro magnetic targeting PTT results, we then researched the in vivo PTT therapeutic effect of the IRFes. When the tumor volume reached $60 \mathrm{~mm}^{3}$, the tumorbearing mice were randomly divided into 5 groups $(n=5)$ : (1) saline; (2) laser irradiation; (3) IRFes; (4) IRFes with laser irradiation; and (5) IRFes with laser irradiation and magnetic targeting. The IRFes were intravenously injected into the tumorbearing mice $\left(200 \mu \mathrm{L}, 10 \mathrm{mg} \mathrm{mL}^{-1}\right)$. As shown in Fig. $7 \mathrm{a}$ and $b$, the infrared thermal images of the IRFes show the results of in vivo PTT. When only under laser irradiation, the local temperature of the tumor did not increase significantly, as the same as the group injected with only saline. In group (3), the temperature did not change remarkably, and the maximum temperature was $27.0 \pm 0.5{ }^{\circ} \mathrm{C}$. The temperature of the other body parts of the mice not exposed to laser irradiation increased negligibly. With prolonged laser irradiation exposure, the surface temperatures of mice tumors in groups (4) and (5) gradually increased, regardless of whether magnet attraction was added or not, and the maximum temperature reached 50.0 $\pm 1.5{ }^{\circ} \mathrm{C}$ and $56.0 \pm 1.1{ }^{\circ} \mathrm{C}$ after $5 \mathrm{~min}$. On the basis of the temperature of the thermal effects, the thermotherapy was divided into warm thermotherapy $\left(40-43^{\circ} \mathrm{C}\right)$ and hyperthermia $\left(43-70{ }^{\circ} \mathrm{C}\right)$ categories. Warm thermotherapy was used throughout the body, and hyperthermia was used locally in the tumor. The experimental results indicated that the magnetic targeting of the IRFes could convert the absorbing NIR light into heat energy, which resulted in the tumor surface temperature increasing to over $50^{\circ} \mathrm{C}$, and this high temperature was limited to the tumor; therefore, the therapy was deemed a local hyperthermia treatment. When the temperature exceeded $43{ }^{\circ} \mathrm{C}$ for only $5 \mathrm{~min}$, tumor cell necrosis was induced. Hence, it was determined that the magnetic targeting IRFes had the ability to
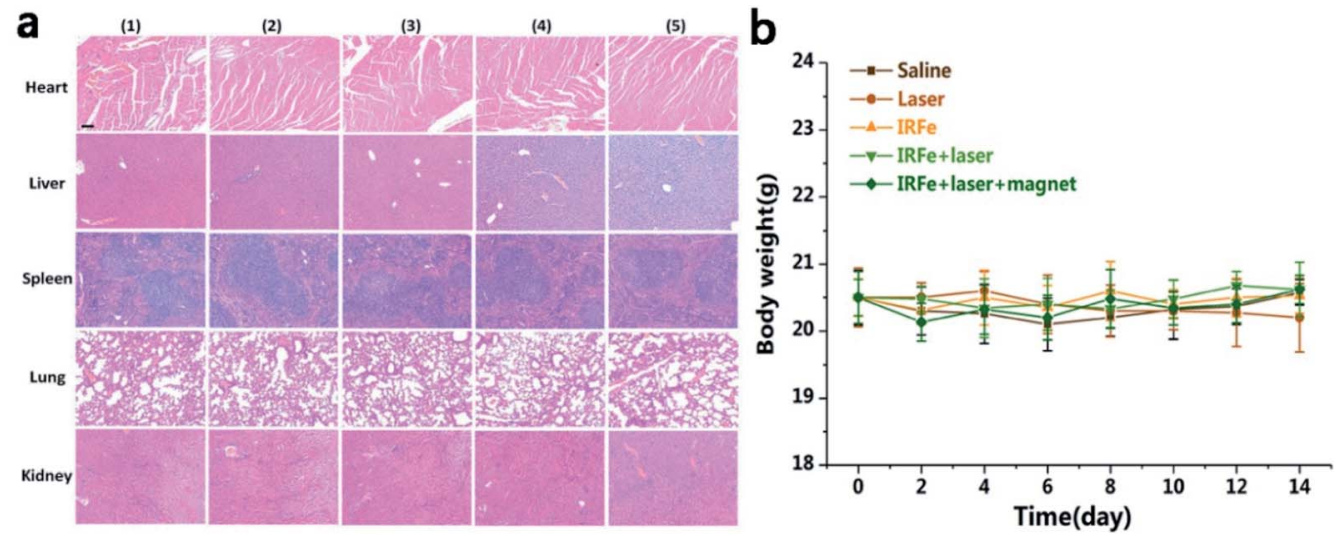

Fig. 6 (a) H\&E staining of heart, liver, spleen, lung, and kidney tissue slices after different treatments: (1) saline, (2) laser, (3) IRFes + laser, (4) IRFes + laser + magnet (scale bar, $50 \mu \mathrm{m}$ ). (b) Changes of mice body weight during different treatments. 

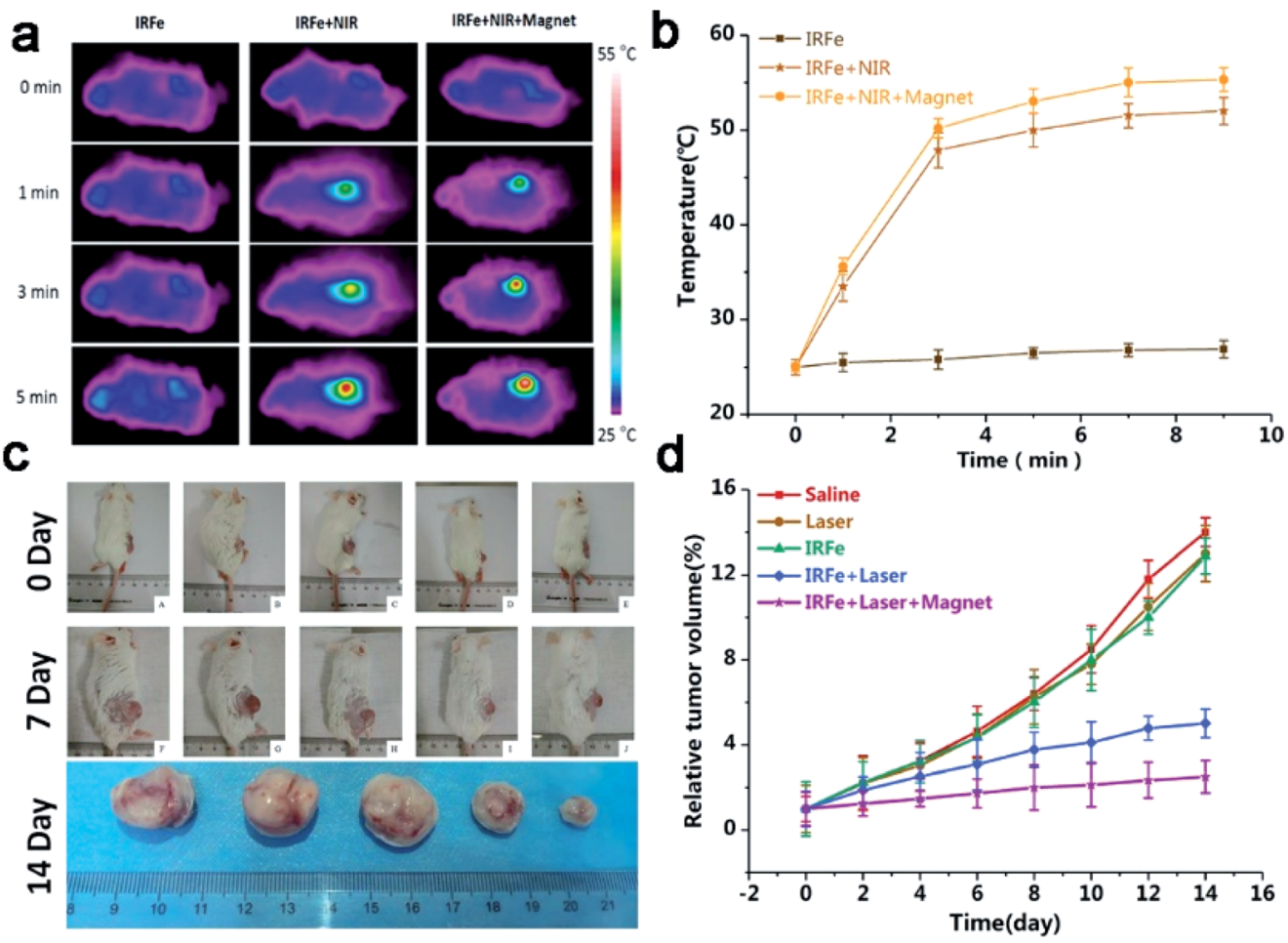

e
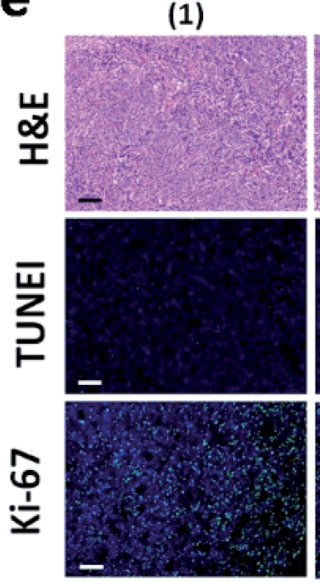

(2)

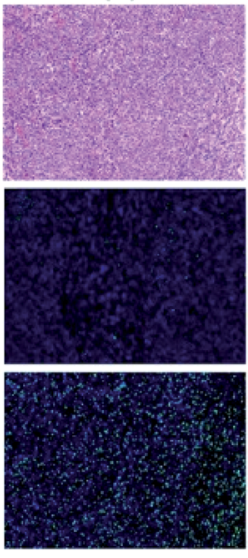

(3)
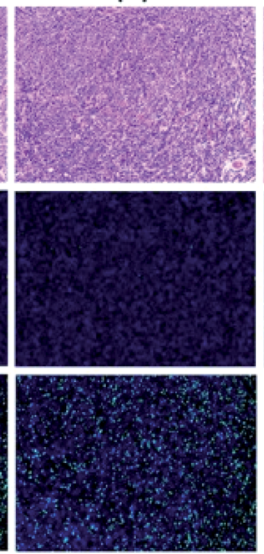

(4)
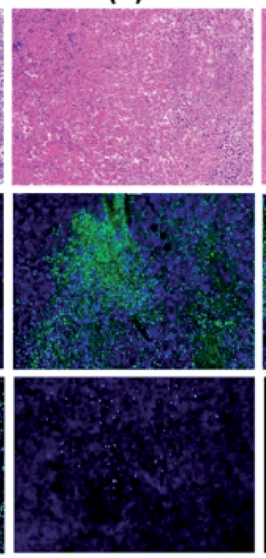

(5)

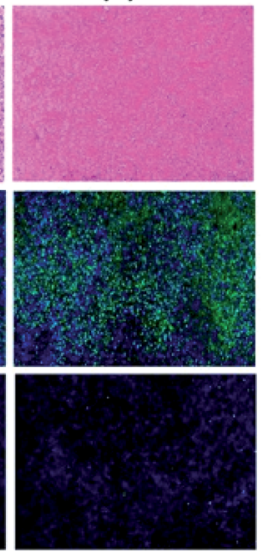

Fig. 7 (a) In vivo antitumor therapy after different treatments $(n=5)$ : (1) saline; (2) laser irradiation; (3) IRFes; (4) IRFes with laser irradiation; (5) IRFes with laser irradiation and magnetic targeting. Infrared thermal images (a) and temperatures changes (b) of tumor-bearing mice treated with IRFes with or without magnetic targeting under laser irradiation ( $808 \mathrm{~nm}, 1 \mathrm{~W} \mathrm{~cm}^{-2}, 10 \mathrm{~min}$ ). (c) The photographs of tumor-bearing mice before and after photothermal therapy at 7 day and ex vivo tumors after photothermal therapy at 14 day. (d) Tumor growth curve after different treatments. (e) H\&E staining, TUNEL and Ki-67 immunofluorescence assays of tumor tissue slices after various treatments (scale bar, $50 \mu \mathrm{m}$ ).

increase the local NP concentration and were splendid photothermal agents in vivo.

Then, we evaluated the antitumor effectiveness of PTT by tumor volume and histological analysis. The tumor sizes were measured every other day after exposure to the treatments. Fig. $7 \mathrm{c}$ and $\mathrm{d}$ shows the changes in mouse tumor volume for the different treatment groups. In the (1) saline-treated group, compared with that of the 0 day injection, the tumor volume increased 13 -fold by day 14 . In addition, it was obvious that the tumor volume and tumor growth rates for groups (2) and (3) increased 12 -fold and 11.88 -fold, respectively, which was a negligible difference, suggesting that neither $808 \mathrm{~nm}$ NIR laser irradiation nor nanoparticles alone suppressed tumor growth. In contrast, the tumors in the mice of group (4) were distinctly small, having increased only 4-fold, showing that the EPR effect of the IRFes also inhibited tumors under $808 \mathrm{~nm}$ laser irradiation. Taken together, these results indicate that IRFes absorb near-infrared light and release a large amount of heat in local tumors, findings that are consistent with the results of the in vitro experiments. Temperature exceeding $42{ }^{\circ} \mathrm{C}$ induced coagulative necrosis of the tumor cells. Most importantly, the tumors in group (5) exposed to IRFes + laser irradiation + magnetic targeting showed the optimal treatment effects: the tumor volume increased relatively approximately 
two-fold because the magnetic targeting led to the accumulation of more IRFes to local tumors and the EPR effect prolonged the retention of these IRFes, thereby enhancing photothermal therapy; this explanation corresponds to the results from the fluorescence imaging experiment. The synergistic effect of both the aggregation caused by the magnetic targeting and the photothermal therapy manifested in low dose IRFes therapy agents with the ability to inhibit tumor growth tremendously. Additionally, the tumor sizes in the mice of group (5), on the 14 th day were much smaller than those of the other groups, as shown in the tumor photographs in Fig. 7c.

The photothermal efficacy was further verified by $\mathrm{H} \& \mathrm{E}$ staining, TUNEL and Ki-67 immunofluorescence assays (Fig. 7e). The H\&E staining observed in the tumor sections further confirmed that the cells in the tumors from the mice injected with the NPs and exposed to $808 \mathrm{~nm}$ laser irradiation were severely damaged, with no structures apparent in the homogeneous red staining, whereas the tumor cells in control groups largely maintained normal morphologies with complete membrane and nuclear structures. The TUNEL assays confirmed that there was more green fluorescence in group (5) cells than was observed for the other groups. The Ki-67 staining of proliferating cells was visualized as green fluorescence, and the results showed that group (5) under $808 \mathrm{~nm}$ laser irradiation and magnetic targeted therapy had the least green fluorescence. The results also showed that magnetic targeted combination therapy could effectively and efficiently suppress the growth of tumor cells and enhance the apoptosis of tumor cells.

The distinct antitumor effect of the IRFes in vivo was due to the following: the magnet led to greater accumulation and treatment application of the IRFes in the tumors, and/or even at low concentrations, the IRFes assimilate near-infrared light rapidly and transform it into enormous heat energy, thereby increasing the cumulative necrosis of tumor cells.

\section{Conclusions}

In summary, a multifunctional nanocomposite, IRFes, with outstanding biocompatibility in a physiological environment was successfully prepared by a simple single emulsion method. Accordingly, these IRFes were used as a novel treatment agent for in vivo fluorescence imaging. Importantly, magnetic targeting of the IRFes more effectively killed cancer cells and enhanced the photothermal therapy that kills cancer cells when exposed to a $808 \mathrm{~nm}$ NIR laser; however, neither the IRFes nor the laser alone significantly influenced cancer cells. This versatile IRFes nanocomposite has tremendous potential for directing the magnetic targeting of PTT by spatially/temporally controlling NIRF imaging. The construction of intelligent IRFes nanotherapeutic agents will open up a new way to efficiently monitor the cancer treatment reaction process and effectively protect surrounding normal tissues from damage.

\section{Conflicts of interest}

The authors declare that they have no conflicts of interest.

\section{Acknowledgements}

This project was funded by the National Natural Science Foundation of China (Grant No. 81974267 and 81601883), Hunan Provincial Natural Science Foundation of China (Grant No. 2018JJ3861) and Hunan Provincial Health Commission Research Foundation Project (B2019166). Animals were performed in accordance with the guidelines of the Department of Laboratory Animals, Central South University, China, and approved by the Ethics Committee of Central South University. Informed consent was obtained from all human participants included in the study.

\section{References}

1 R. L. Siegel, K. D. Miller and A. Jemal, Ca-Cancer J. Clin., 2016, 66, 7-30.

2 M. S. Tessmer and K. T. Flaherty, Clin. Cancer Res., 2017, 23, 5325.

3 K. D. Miller, R. L. Siegel, C. C. Lin, A. B. Mariotto, J. L. Kramer, J. H. Rowland, K. D. Stein, R. Alteri and A. Jemal, Ca-Cancer J. Clin., 2016, 66, 271-289.

4 R. J. Bose, R. Paulmurugan, J. Moon, S. H. Lee and H. Park, Drug Discovery Today, 2018, 23, 891-899.

5 S. Mitragotri, P. A. Burke and R. Langer, Nat. Rev. Drug Discovery, 2014, 13, 655-672.

6 B. Chertok, M. J. Webber, M. D. Succi and R. Langer, Mol. Pharm., 2013, 10, 3531-3543.

7 D. Peer, J. M. Karp, S. Hong, O. C. Farokhzad, R. Margalit and R. Langer, Nat. Nanotechnol., 2007, 2, 751-760.

8 L. R. Hirsch, R. J. Stafford, J. A. Bankson, S. R. Sershen, B. Rivera, R. E. Price, J. D. Hazle, N. J. Halas and J. L. West, Proc. Natl. Acad. Sci. U. S. A., 2003, 100, 13549-13554.

9 K. F. Chu and D. E. Dupuy, Nat. Rev. Cancer, 2014, 14, 199208.

10 J. Nam, S. Son, L. J. Ochyl, R. Kuai, A. Schwendeman and J. J. Moon, Nat. Commun., 2018, 9, 1074.

11 H. Liu, D. Chen, L. Li, T. Liu, L. Tan, X. Wu and F. Tang, Angew. Chem., Int. Ed. Engl., 2011, 50, 891-895.

12 Z. Zhang, J. Wang, X. Nie, T. Wen, Y. Ji, X. Wu, Y. Zhao and C. Chen, J. Am. Chem. Soc., 2014, 136, 7317-7326.

13 M. P. Melancon, M. Zhou and C. Li, Acc. Chem. Res., 2011, 44, 947-956.

14 L. Cheng, H. Gong, W. Zhu, J. Liu, X. Wang, G. Liu and Z. Liu, Biomaterials, 2014, 35, 9844-9852.

15 C. Yue, P. Liu, M. Zheng, P. Zhao, Y. Wang, Y. Ma and L. Cai, Biomaterials, 2013, 34, 6853-6861.

16 J. Liu, X. Zheng, L. Yan, L. Zhou, G. Tian, W. Yin, L. Wang, Y. Liu, Z. Hu, Z. Gu, C. Chen and Y. Zhao, ACS Nano, 2015, 9, 696-707.

17 J. Yu, W. Yin, X. Zheng, G. Tian, X. Zhang, T. Bao, X. Dong, Z. Wang, Z. Gu, X. Ma and Y. Zhao, Theranostics, 2015, 5, 931-945.

18 G. S. Gazelle, S. N. Goldberg, L. Solbiati and T. Livraghi, Radiology, 2000, 217, 633-646.

19 S. N. Goldberg, Eur. J. Ultrasound, 2001, 13, 129-147. 
20 F. Wu, W. Z. Chen, J. Bai, J. Z. Zou, Z. L. Wang, H. Zhu and Z. B. Wang, Ultrasound Med. Biol., 2001, 27, 1099-1106.

21 Y. Liu, H. Miyoshi and M. Nakamura, Int. J. Cancer, 2007, 120, 2527-2537.

22 D. de Melo-Diogo, C. Pais-Silva, D. R. Dias, A. F. Moreira and I. J. Correia, Adv. Healthc. Mater., 2017, 6, 1700073.

23 P. Zhang, L. Zhang, Z. Qin, S. Hua, Z. Guo, C. Chu, H. Lin, Y. Zhang, W. Li, X. Zhang, X. Chen and G. Liu, Adv. Mater., 2018, 30, 1705350.

24 C. Pais-Silva, D. de Melo-Diogo and I. J. Correia, Eur. J. Pharm. Biopharm., 2017, 113, 108-117.

25 C. Jiang, H. Cheng, A. Yuan, X. Tang, J. Wu and Y. Hu, Acta Biomater., 2015, 14, 61-69.

26 F. Guo, M. Yu, J. Wang, F. Tan and N. Li, ACS Appl. Mater. Interfaces, 2015, 7, 20556-20567.
27 H. Choi, S. R. Choi, R. Zhou, H. F. Kung and I. W. Chen, Acad. Radiol., 2004, 11, 996-1004.

28 C. Niu, Z. Wang, G. Zuo, T. M. Krupka, H. Ran, P. Zhang, P. Li, Y. Chen, H. Chen and Y. Zheng, Clin. Breast Canc., 2012, 12, 199-206.

29 Y. Chen, Z. Li, H. Wang, Y. Wang, H. Han, Q. Jin and J. Ji, ACS Appl. Mater. Interfaces, 2016, 8, 6852-6858.

30 L. Wang, S. Chen, Y. Zhu, M. Zhang, S. Tang, J. Li, W. Pei, B. Huang and C. Niu, ACS Appl. Mater. Interfaces, 2018, 10, 42102-42114.

31 J. Peng, T. Qi, J. Liao, B. Chu, Q. Yang, Y. Qu, W. Li, H. Li, F. Luo and Z. Qian, Theranostics, 2014, 4, 678-692.

32 M. Li, W. Bu, J. Ren, J. Li, L. Deng, M. Gao, X. Gao and P. Wang, Theranostics, 2018, 8, 693-709. 